\title{
Spectrophotometric Determination of Trimebutine Through lon- Pair and Charge- Transfer Complexation Reactions
}

\author{
Shaban M. Khalil \\ National Organization for Drug Control and Research \\ P.O. Box 29 Cairo (NODCAR) Egypt
}

Summary Two simple and sensitive spectrophotometric methods are described for the assay of trimebutine. The first method is based on the interaction of the basic drug in 1,2 dichloroethane with bromocresol green (BCG); bromophenol blue (BPB) and bromothymol blue (BTB) in the same solvent to produce a stable yellow ion-pair complexes, which absorbed at 410,406 and $405 \mathrm{~nm}$, respectively. The second method is based upon the interaction of the basic drug with iodine in 1,2 dichloroethane. The yellow colour formed due to the formation of charge-transfer complex showed two maxima absorbed at 292 and $362 \mathrm{~nm}$. Beer's law obeyed for both methods and the relative standard deviations were found to be less than $1 \%$. The two methods can be applied to the analysis of tablets, with no evidence of interference from excipients. A more detailed investigation on the complex was made to its composition, association constant and free energy change.

Keyword : Spectrophotometry ; charge - transfer and ion - pair complexes; tablets.

\section{Introduction}

Trimebutine, 2-dimethylamino-2-phenylbutyl1, 3, 4, 5 trimethoxybenzoate, is an antispasmodic compound used in various gastrointestinal diseases 
and in radiological examination ${ }^{(1)} . \operatorname{HPLC}^{(2,3)}$, and colourimetric ${ }^{(4)}$ methods for its assay have been used. Methods for determination of the drug in pharmaceutical preparations are not yet available in the literature or in pharmacopoeias. In the present paper simple and sensitive spectrophotometric methods for determining the drug, based on the formation of ion-pair with BCG, BPB, BTB and charge-transfer with $I_{2}$ in 1,2dichloroethane were proposed.The proposed methods have been applied to assay of the drug in the tablet.

\section{Experimental}

Apparatus : A Shimadzu 160A UV/visible double beem spectrophotometer with matchedquartz cells of 1-cm optical path was used.

Materials Trimebutine (Roussel Uclaf, France) was used as working standard. Debridat tablet $100 \mathrm{mg} / \mathrm{tab}$. (Hoechst Orient Egypt Under Licence of Roussel Uclaf, France).

Reagents All the reagents and solvents used were of analytical grade. BCG (Koch-Light Laboratories Ltd), BPB and (Aldrich Chemical Co. Ltd), and 12 (acf chemie farma nv, maarssen-- holland). BCG, BPB, BTB

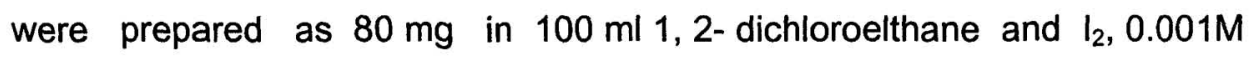
in 1, 2 dichloroelthane. The iodine solution must be protected from light.

Preparation of standard solution Trimebutine stock solution was prepared by dissolving $10 \mathrm{mg}$ of trimebutine in $25 \mathrm{ml}$ of 1,2-dichloroelthane.

Construction of calibration curves: Aliquots of a solution of the drug (in concentration range cited in table1) were transferred into $10 \mathrm{ml}$ measuring flasks. To each flask, $1 \mathrm{ml}$ of BCG, BPB, BTB or $\mathrm{I}_{2}$ solution was added, mixed well and allowed to stand at room temperature $\left(25 \pm 1^{\circ} \mathrm{C}\right)$ for 10 minutes. The solution was diluted to volume with 1,2 dichloroethane. The absorbances of the resulting complexes were measured at $410,406,405$ and 
$362 \mathrm{~nm}$ for BCG, BPB, BTB and $\mathrm{I}_{2}$ complexes, respectively, against a reagent blank similarly prepared. In case of iodine complex the procedure must be done in dark place.

\section{Analysis of Debridat tablet}

An accurately weighed amounts powdered tablets equivalent to $40 \mathrm{mg}$ of trimebutine base was transferred into a $100 \mathrm{ml}$ separating funnel, and mixed with $20 \mathrm{ml}$ of $0.1 \mathrm{M}$ potassium hydroxide and shaking with five $20 \mathrm{ml}$ portions of 1,2 dichloroethane. The combined extracts washed by distilled water, dried with anhydrous sodium sulphate for 5 minutes, filtered through dry filter paper into a $100 \mathrm{ml}$ measuring flask, and completed to the volume with the same solvent if necessary. Then procedure can be proceeded as described under "construction of calibration curves "

\section{Stoichiometric relationship}

Job's method of continuous variation of equimolar solutions was employed: a $1 \times 10^{-3} \mathrm{M}$ of standard solution of drug and $1 \times 10^{-3} \mathrm{M}$ of each $B C G, B P B$, and BTB solutions were used but in case of $I_{2} 2 \times 10^{-3}$ was used. A series of drug and reagent solutions was prepared in which the total volume of drug and reagent was kept at $1 \mathrm{ml}$. The reaction solutions were mixed well and allowed to stand for 10 minutes at $25 \pm 1{ }^{\circ} \mathrm{C}$. The solutions were diluted to $10 \mathrm{ml}$ with 1,2 - dichloroethane and the absorbance was measured at the specific wavelength mentioned before.

\section{Association constant and free energy}

Serial volumes of $0.2-1.0 \mathrm{ml}$ of $1 \times 10^{-3} \mathrm{M}$ trimebutine solution were transferred to $10-\mathrm{ml}$ standard flasks. To each flasks, $0.15 \mathrm{ml}$ of $1 \times 10^{-3} \mathrm{M}$ of $\mathrm{BPB}$ or $\mathrm{I}_{2}$ was added continued as directed under construction of calibration curves. 


\section{Results and discussion}

Ion pair method: The absorption spectra of the ion-pair complexes formed between trimebutine or $\mathrm{BCG}, \mathrm{BPB}$, or $\mathrm{BTB}$ were measured in the range 300- $500 \mathrm{~nm}$ against the blank reagent (Fig.1) which exhibit a broad absorption maximum at 410,406 and $405 \mathrm{~nm}$ for BCG, BPB and BTB ion pair complexes, respectively. These dyes belong to the family of sulphonephthalin dyes, and have different ionic forms. The yellow spices HL are present in aqueous solutions. On acidification, they can turn purple $\left(\mathrm{H}_{2} \mathrm{~L}\right.$ as an ampholyte ); this colour change corresponds to protonation of the quinoid oxygen. The second symmetric resonance structure $\left(L^{2}\right)$ arises by splitting of a proton from the hydroxy group, accompanied by bathochromic shift ${ }^{(5)}$. Since the reaction product in 1,2 dichloroethan is yellow, so, it may be considered that $\mathrm{HL}$ is the form of the BCG, BPB or $B T B$ involved in the reaction with the chosen drug. This may lead to an assumption that, by the interaction of BCG, BPB, and BTB to the basic center of the drug takes place. The obtained ion-pair salt is dissociated to the yellow $\mathrm{HL}$ anion.

CT method: The reaction of $I_{2}$ with basic nitrogenated drugs results in the formation of an intense yellow colour product which exhibits absorption maxima at 292 and $362 \mathrm{~nm}$ (Fig.1).

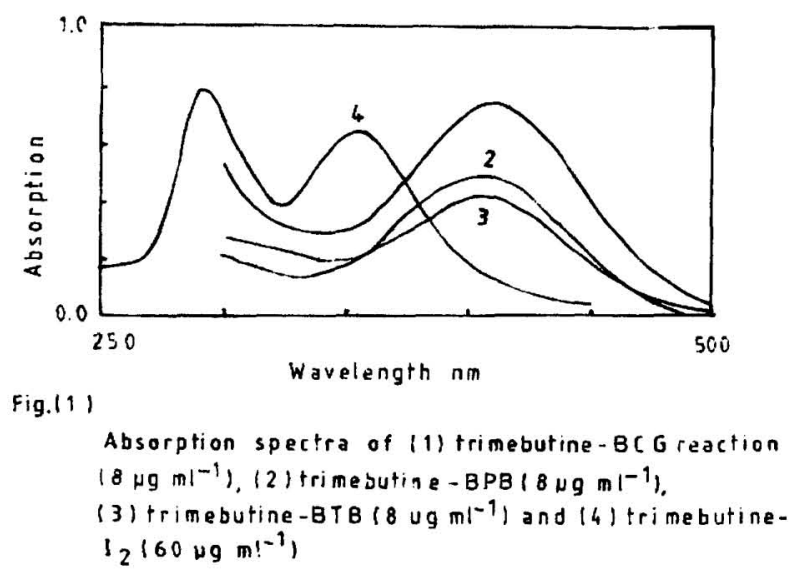


These bands may be attributed to the formation of the iodine radical anion. As described in the literature ${ }^{(6)}$ the formation of $I_{3}$ anion which is measurable spices, is due to the transformation of an " outer complex" to an " inner complex "liberating $I^{-}$ions which react with the free molecular iodine. In other words, the interaction between trimebutine and iodine is a charge-transfer complexation reaction between $n$-donor and the $\delta$-acceptor iodine followed by the formation of a radical ion according to the following scheme.

$$
\begin{aligned}
& \mathrm{D}+\mathrm{I}_{2} \longrightarrow \mathrm{D}-\mathrm{I}_{2} \quad \begin{array}{l}
\text { (outer complex) } \\
\mathrm{D}-\mathrm{I}_{2} \longrightarrow\left[\mathrm{D}-\mathrm{I}^{+} \mathrm{I}^{-}\right] \quad(\text { inner complex) } \\
{[\mathrm{D}-\mathrm{I}]^{+} \mathrm{I}^{-}+\mathrm{I}_{2} \longrightarrow}
\end{array}
\end{aligned}
$$

where $D$ is the drug under investigation.

Regarding the third step in the above scheme, iodine alone does not absorb at the wavelength of the maximum absorption, hence the stoichiometry will show only the iodine ion released in the second step as a result of one mole of iodine being consumed in the third step. Although the complex was formed rapidly,constant absorbance reading were obtained after10 minutes on standing in the dark at $25 \pm 1^{\circ} \mathrm{C}$, and remained constant for more than $24 \mathrm{~h}$, i.e. the complex is stable.

The effect of solvent on the formation of ion - pair complexes were studied using acetonitrile, 1, 2- dichloroethane, dichloromethane and chloroform. The 1,2-dichloroethane was preferred because of the higher molar absorptivities and stabilities of the complexes formed in it. The same solvent was considered to be an ideal solvent for the colour reaction and it offers excellent solvent capacity for iodine CT complex and gives the highest yield of the radical. The effect of reagents concenteration has been studied. It was found that, $0.4 \mathrm{ml}$ of $\left(400 \mu \mathrm{g} \mathrm{ml}^{-1}\right)$ of BCG, BPB 
was required for maximum complex formation [Fig.2], and $1 \mathrm{ml}$ of $1 \times 10^{-3} \mathrm{M}$ $\mathrm{I}_{2}$ was enough to give maximum absorption of the CT complex found. The excess of reagents has no effect

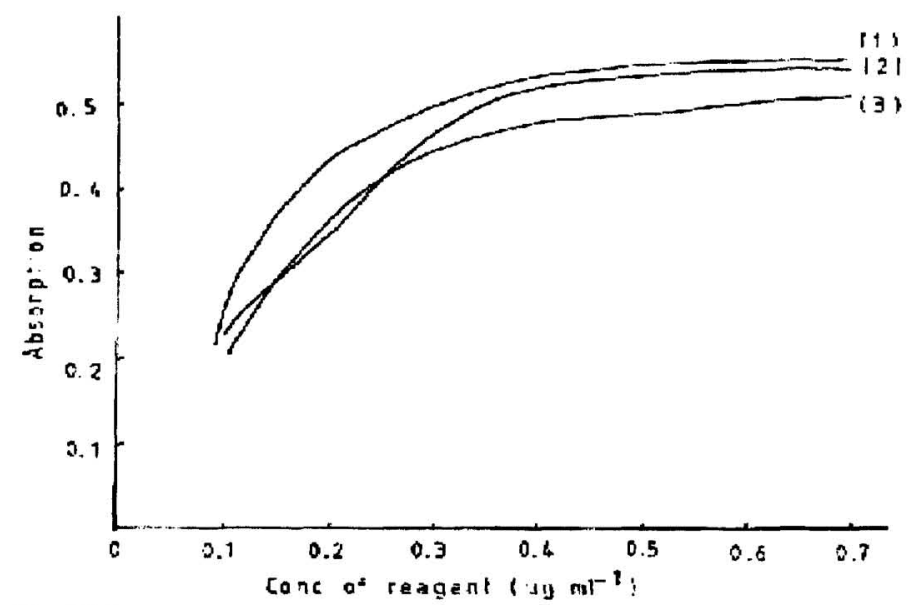

Fig.12,

Effect of reagent conepntration of (1) BT3,121BPB,

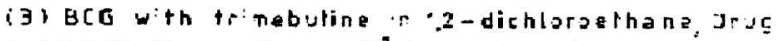

concentration $=8 \mathrm{pg} \mathrm{m:}^{-1}$ in each instarce.

The molar ratio of the reactants (Drug: reagent) in the charge - transfer or lon - pair complex was determined by the method of continuous variation (Job's method) and found to be $1: 1$ [Fig. 3]. This finding was anticipated by the presence of one basic or electron - donating center in the drug studied. 


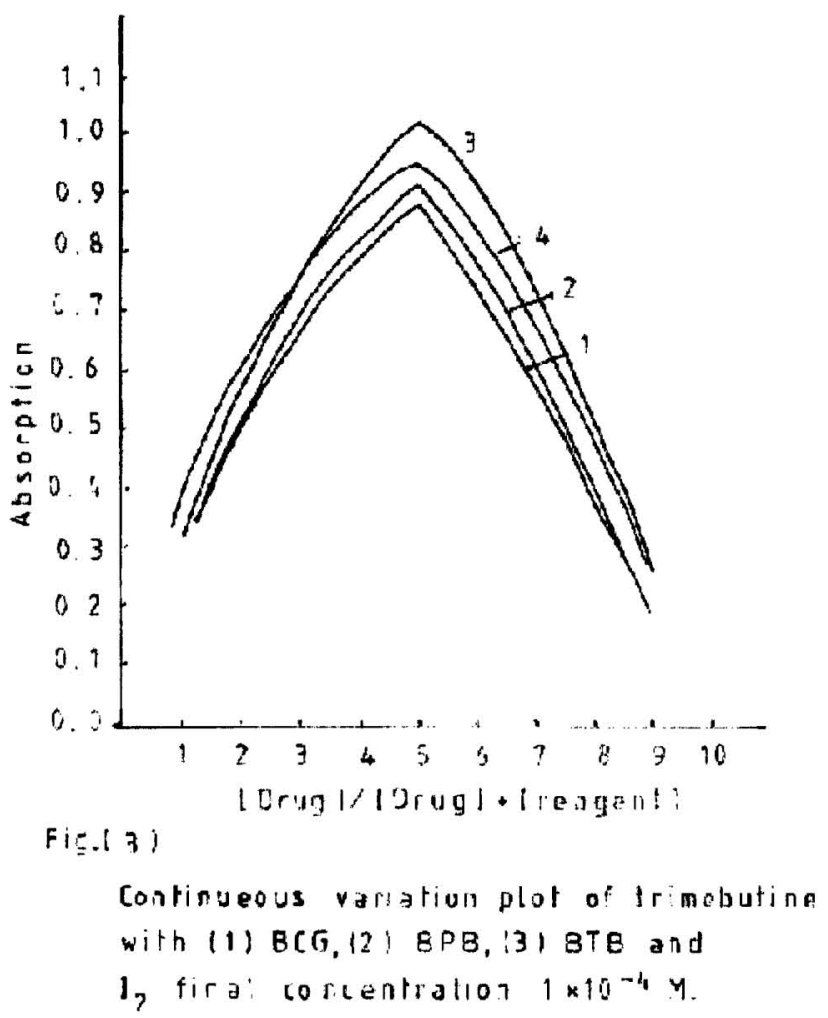

A more detailed examination was made for the drug - BPB or the drug $I_{2}$ complex. The absorbance of the complex was used to calculate the association constant using the Benesi - Hildebrand equation ${ }^{(7)}$.

$\frac{[A 0]}{A_{\lambda}^{A D}}=\frac{1}{E_{\lambda}^{A D}}+\frac{1}{K C^{A D} E \lambda}{ }_{A D} \times \frac{1}{[D 0]}$

Where $[A 0]$ and $[D 0]$ are the total concentration of the interacting species, $A \Lambda^{A D}$ and $E \lambda^{A D}$ are the absorbance and the molar absorptivity of the complex at 406 constant of the complex. On plotting the values of $[A 0] / A \lambda^{A D}$ vs $1 /[D 0]$, a line was obtained (Fig.4,5) that is described by the following equation: 
[A0]

1 $\frac{\left.A_{0}\right]}{A_{\Lambda}^{A D}}=0.375 \times 10^{-4}+0.031 \times 10^{-8}$

(2) (for BPB complex)

[Ao]

$=0.325 \times 10^{-4}+0.204 \times 10^{-8}$

1

$A_{\lambda}^{A D}$ [D0]

(3) (for $\mathrm{I}_{2}$ complex)

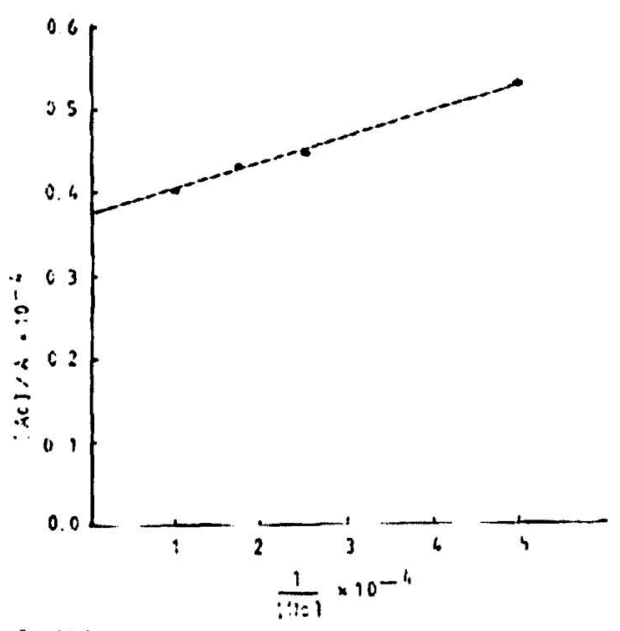

$F(g(6)$

Benes- inldeorand plot fur trimesuline-Bpt complex. $\lambda=606 \mathrm{~nm}$.

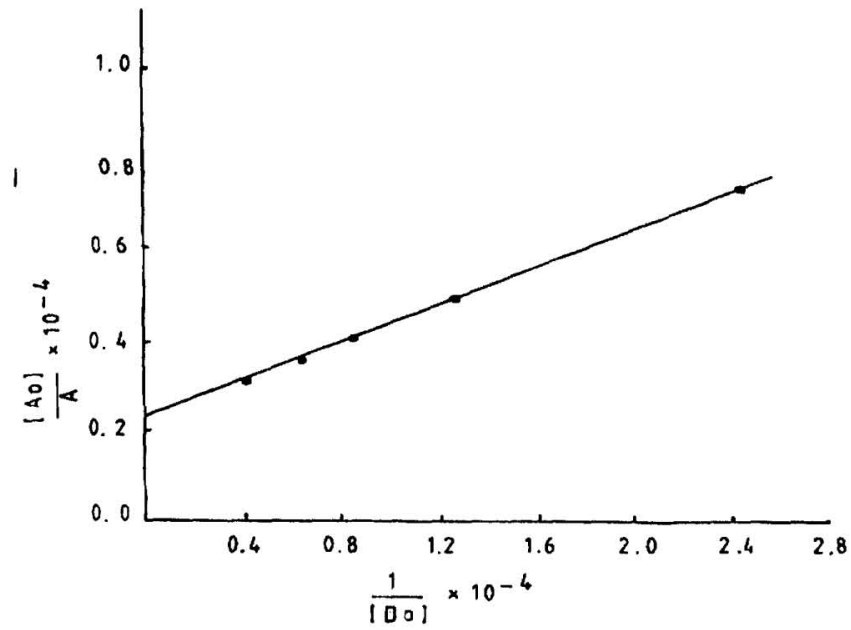

Fig.(5)

Benesi-Hildebrand plot for trimebutine-l complex. $\lambda=362 \mathrm{~nm}$ 
The intercept of this line with the ordinate is $(E \lambda) A D^{-1}$, the slope equals $\left(E_{\lambda}{ }^{A D} K C^{A D}\right)^{-1}$. From the equation (2) and (3) the association constants are $1.21 \times 10^{5}$ and $1.15 \times 10^{4} \mathrm{~L}^{\mathrm{mol}}{ }^{-1}$ and the standard free energies of complexation $\Delta G^{\circ}$ are -6.976 and $-5.572 \mathrm{~K}$. cal. For BPB and $\mathrm{I}_{2}$ complexes, respectively. The molar absorptivities are equal to $26.1 \times 10^{3}$ and $42.5 \times 10^{3} \mathrm{~L} \mathrm{~mol}^{-1} \mathrm{~cm}^{-1}$ for BPB and $I_{2}$ - trimebutine complexes respectively. which are comparable with those obtained from the regression line equation of Beer's Law (table 1).

Table (1) Quantitative parameters for the complexation of trimebutine with BCG, BPB, BTB and $\mathrm{I}_{2}$

\begin{tabular}{|c|c|c|c|c|}
\hline Parameters & BCG & BPB & BTB & $\mathrm{I}_{2}$ \\
\hline Beer's Law limits $\left(\mu \mathrm{g} \cdot \mathrm{ml}^{-1}\right)$ & $1-10$ & $1-8$ & $1-10$ & $1-24$ \\
Molar absorptivity $\left(1 . \mathrm{mol}^{-1}\right.$ & $17.79 \times 10^{3}$ & $26.21 \times 10^{3}$ & $19.548 \times 10^{3}$ & $42.036 \times 10^{3}$ \\
$\left.\mathrm{~cm}^{-1}\right)$ & & & & \\
Sandell sensitivity $\left(\mu \mathrm{gg} . \mathrm{cm}^{-2}\right)$ & $21.8 \times 10^{-3}$ & $14.8 \times 10^{-3}$ & $20.8 \times 10^{-3}$ & $62.44 \times 10^{-3}$ \\
Slope $^{*}$ & 0.048 & 0.0678 & 0.054 & 0.0157 \\
Intercept* & 0.0132 & 0.0002 & 0.0308 & 0.0066 \\
${\text { Correlation coefficient }{ }^{*}}_{\text {Standard error }}^{0.9966}$ & 0.9989 & 0.9993 & 0.9985 \\
& 0.0144 & 0.0117 & 0.0074 & 0.0072 \\
& & & & \\
\hline
\end{tabular}

${ }^{*} n=5$ all instances.

Quantification, accuracy and precision: The reproducibility and accuracy of the suggested methods were assessed using different concentrations. The validity was checked occasionally during the work by assaying standards. Calibration curves for trimebutine was prepared by taking series of different concentrations and applying the suggested procedures with BCG, BPB,BTB and $I_{2}$. Beer's Law is valid within microgram concentration range of trimebutine (table1)for each reagent. The regression equation of these calibration graphs were utilized or determination of unknown concentration of 
trimebutine in tablets. The results obtained were of good accuracy and precision. The produced results were reproducible with low standard deviations (table2).

Table (2) Statistical analysis of results obtained for tablets

Proposed methods.

\begin{tabular}{|l|c|c|c|l|}
\hline \multicolumn{1}{|c|}{ Statistic } & BCG & BPB & BTB & $\mathrm{I}_{2}$ \\
\hline Mean \% recovery & 98.24 & 98.18 & 98.05 & 98.22 \\
\pm S. D. & 09.0026 & 0.1109 & 0.1033 & 0.0537 \\
$\mathrm{~N}$ & 5 & 5 & 5 & 5 \\
Variance & 0.00001 & 0.0123 & 0.1068 & 0.002 \\
\hline
\end{tabular}

Table (3) Statistical analysis of results obtained using the proposed methods and reference method [8] for analysis of authentic sample.

\begin{tabular}{|c|c|c|c|c|c|}
\hline Statistic & Reference method & BCG & BPB & BTB & $\mathrm{I}_{2}$ \\
\hline $\begin{array}{c}\text { Mean \% recovery } \\
(p=5)\end{array}$ & 98.00 & 99.706 & 97.09 & 97.00 & 98.67 \\
\pm S. D. & & & & & \\
$N$ & 0.0003 & 0.030 & 0.074 & 0.075 & 0.035 \\
Variance & 5 & 5 & 5 & 5 & 5 \\
$t$ - test & & 0.0009 & 0.0055 & 0.0056 & 0.0012 \\
F - test & & 3.000 & 18.333 & 18.666 & 4.000 \\
& & 3.652 & 5.094 & 2.049 & 3.308 \\
\hline
\end{tabular}

Values of parentheses are the tabulated values of $t$ and $f$ at $p=5$

For comparison, the non-aqueous titrimetric method ${ }^{(8)}$ (based on the titration of trimebutine dissolved in glacial acetic acid with perchloric acid) was applied. The results agreed well with those of the suggested methods since the calculated $\mathrm{t}$ - and $\mathrm{F}$ - values did not exceed the theoretical values (table3).

The non - aqueous method required high concentration of the drug to permit the titrimetric process, in comparison with the suggested methods 
which applied successfully for microgram amounts. The methods described are simple and sensitive. No interference was found due to the foreign excipients.The proposed methods can be used as a control methods for production lots.

\section{References}

(1) M.F. Moshal , j. Int. Med. Res., 7 (1979) 232.

(2) A. Astier and A. M. Deutsh, J. Chromatogr. , 224 (1981) 149.

(3) E. H. Joo, W. I. Chab , I. Oh, S.C. Shin, H. K. Na, Y.B .Lee,J.

Chromatogr, -B, Biomed-Appl. 723(1-2) (1999) $239-246$.

(4) F.M. Abdel-Gawad, J. Pharm-Biomed-Anal 16 (5) (1998)793-799.

(5) K. Vytras, H. Batova and . Janousek. Analyst 114 (1989) $479-483$

(6) A.I. Popov and W. Deskin; J.Am. Chem.Soc. 80, (1958) 2976-2979

(7) H.A. Benesi and J. Hildebrand, J.Am. Chem. Soc.71(1949)2703 2707.

(8) The specifications and quality control of Roussel Uclaf company. 